\title{
Chef- und Leitende Ärztinnen und Ärzte im Spannungsfeld des neuen Arbeitsgesetzes (ArG)
}

\author{
U. Reinhard
}

1 Reinhard U. Anwendbarkeit des Arbeitsgesetzes (ArG) auf Spitäler und Spitalärzte. Schweiz Ärztezeitung 2005;86(19):1137-9.

a Die angegebenen Stundenzahlen gelten nur als Grundsätze; je nach Betrieb und Organisation des Spitals sind verschiedenste Ausnahmen möglich.

b Vgl. Art. 59 ff. ArG, im Internet zu finden unter www.admin.ch/ ch/d/sr/c822_11.html.

Korrespondenz:

lic. iur. Urs Reinhard Rechtsberatungsstelle

Verein der Leitenden Spitalärzte

der Schweiz VLSS

Gutenbergstrasse 9

CH-3011 Bern
Auf den 1. Januar 2005 haben das Arbeitsgesetz und einige seiner Verordnungen Änderungen erfahren, die auf die Anstellungsverhältnisse von Spitalärztinnen und Spitalärzten nicht ohne Einfluss bleiben werden. Während in einer früheren Ausgabe [1] dargelegt wurde, ob und wie die einzelnen Ärztinnen und Ärzte an den Spitälern vom Arbeitsgesetz erfasst werden, soll nun ein Problem beleuchtet werden, das sich mit der Unterstellung der Assistenzärztinnen und -ärzte unter das ArG den meisten Chef- und Leitenden Ärztinnen und Ärzten stellen wird.

\section{Erfassung aller Assistenzärztinnen und -ärzte durch das revidierte Arbeitsgesetz}

Assistenzärztinnen und -ärzte waren bis Ende des letzten Jahres vom Geltungsbereich des ArG ausgenommen. Auf den 1. Januar 2005 traten jedoch zahlreiche Gesetzes- und Verordnungsänderungen in Kraft, womit Assistenzärztinnen und -ärzte ausdrücklich als vom ArG erfasst bezeichnet werden: Alle Assistenzärztinnen und -ärzte, sowohl diejenigen privater als auch öffentlich-rechtlicher Spitäler, ungeachtet deren Rechtsform, sind dem ArG nun vollumfänglich unterstellt. Dies hat zur Folge, dass für sie die strengen Regeln zu Arbeits- und Ruhezeiten gelten: Die wöchentliche Höchstarbeitszeit beträgt 50 Stunden, die maximale Arbeitszeit pro Tag 14 Stunden, die tägliche Ruhezeit muss 11 Stunden (aufeinanderfolgend) betragen, Nachtarbeit darf höchstens 12 Stunden dauern, ohne geeignete vorhandene Ruhegelegenheit nur 10 Stunden ${ }^{\mathrm{a}}$.

\section{Mögliche Auswirkungen auf die Ausgestaltung der Dienstpläne}

Wenn diese stundenmässigen Beschränkungen für die betroffenen Assistenzärztinnen und -ärzte auch erfreulich sein mögen, so stellen sie die Chef- und die Leitenden Ärztinnen und Ärzte doch vor grosse Schwierigkeiten: Die Dienstpläne können angesichts der beschriebenen ein- geschränkten Einsetzbarkeit der Assistenten z.T. nicht mehr so gestaltet werden, dass den Bedürfnissen des Spitals und der Patienten Genüge getan würde. Dies stellt den einteilenden Arzt vor die Wahl, entweder die Vorgaben des ArG strikte zu befolgen und in Verletzung seiner Dienstpflichten den Dienstplan so zu gestalten, dass dieser einen ordnungsgemässen Spitalbetrieb nicht zulässt, oder aber die Einteilung den Bedürfnissen des Spitals gemäss vorzunehmen und dabei zu riskieren, sich strafbar zu machen, denn: Die Verletzung des Arbeitsgesetzes zieht Gefängnis bis zu sechs Monaten oder Busse nach sich $^{b}$.

\section{Rechtsverwahrung}

Dass nur die zweite Variante, nämlich die Ausgestaltung des Dienstplanes nach den Bedürfnissen des Spitals und insbesondere der Patienten, einen gangbaren Weg darstellt, leuchtet ein und ist unser tiefstes ärztliches Selbstverständnis. Allerdings soll der einteilende Arzt nicht - in gefährlich sprichwörtlicher Weise! - dafür büssen, dass er dies allenfalls nur unter Verletzung des ArG tun kann. Der VLSS schlägt deshalb vor, dass sich die Chef- und Leitenden Ärztinnen und Ärzte mittels einer Rechtsverwahrung zuhanden der Spitalleitung gegen allfällige strafrechtliche Konsequenzen schützen. Sie kann beispielsweise folgenden Inhalts sein:

«Aufgrund der Tatsache, dass wir an unserer Klinik/Abteilung die verlangten organisatorischen Optimierungen der Betriebsabläufe umgesetzt haben, situative Controllingmechanismen installiert worden sind, der zusätzliche Bedarf an Personal im ärztlichen Dienst (OA, Assistenten) gemessen und dokumentiert worden ist und fundierte Gesuche um entsprechende personelle Mittel form- und inhaltgerecht eingereicht worden sind, lehne ich/lehnen wir jede persönliche rechtliche Konsequenz im Sinne der Rechtsverwahrung für Verletzungen des Arbeitsgesetzes bzw. des Gesamtarbeitsver- 
c Art. 34 StGB: «Die Tat, die jemand begeht, um das Gut eines andern, namentlich Leben, Leib, Freiheit, Ehre, Vermögen, aus einer unmittelbaren, nicht anders abwendbaren Gefahr zu erretten, ist straflos. [...].»

d ZR 87 (1988) S. 31 (Nr. 13). trages ab, sollten unsere Anträge für zusätzliche Ressourcen zur Erfüllung des Arbeitsgesetzes bzw. des GAV von den zuständigen Stellen abschlägig beantwortet werden.»

Unterschrift/en

\section{Notstand}

Das Dilemma, dass der einteilende Arzt das ArG wenn überhaupt ja nur unverschuldet verletzt, weil er selber nicht für die mangelnden Ressourcen verantwortlich gemacht werden kann, ist dem Strafrecht bekannt. Das Institut des Notstands, Art. 34 des Strafgesetzbuches [StGB], trägt solchen Fällen Rechnung, wo ein «Täter» gar nicht anders kann, als gesetzeswidrig zu handeln, weil ihn gleichzeitig eine Handlungspflicht (hier: Aufrechterhaltung des Spitalbetriebes) und eine Unterlassungspflicht (hier: Nichtverletzung des ArG) treffen, die er unmöglich beide befolgen kann. Die Folge der Anwendbarkeit von Art. 34 StGB ist Straflosigkeit für die handelnde Person ${ }^{\mathrm{c}}$.

In einem Entscheid aus dem Kanton Zürich wurde der Verantwortliche für den EDV-Betrieb einer Bank (was wohl dem Verantwortlichen für einen Teilbereich eines Spitals entsprechen dürfte) trotz mehrfacher Verletzung des ArG freigesprochen. Er liess dringend notwendige Arbei- ten für die Umstellung des Betriebssystems einer Bank v.a. nachts ausführen, um das Tagesgeschäft der Bank zu schonen, und verletzte dabei die Arbeits- und Ruhezeitvorschriften für seine Angestellten. Das Gericht sah Notstand als gegeben an, da es «nicht zumutbar war, den guten Ruf und das Vermögen der Bank preiszugeben», was bei Durchführung der Arbeiten gemäss den Vorschriften des ArG gedroht hätte ${ }^{\mathrm{d}}$. Wenn der gute Ruf und das Vermögen einer Bank als Gründe ausreichen, um das ArG verletzen zu dürfen, so muss dies um so mehr gelten, wenn die Gesundheit von Patientinnen und Patienten auf dem Spiel steht.

\section{Fazit}

Chef- und Leitende Ärztinnen und Ärzte, die bei der Gestaltung des Dienstplans gezwungenermassen das ArG verletzen, kommen in den Genuss der Anwendung des Art. 34 StGB, welcher Straflosigkeit vorsieht. Damit Leitende Spitalärztinnen und -ärzte aber unabhängig von einer korrekten Rechtsanwendung durch den Richter (oder vorzugsweise ohne einen Richter überhaupt anrufen zu müssen) den Dienstplan gefahrlos nach den Bedürfnissen des Spitals und der Patienten festlegen können, ist das Verfassen einer Rechtsverwahrung zuhanden der Spitalleitung dringend angezeigt. 